\title{
Nonlinear coded nonuniform superposition QAM by trellis-coding for MISO system in visible light communication
}

\author{
Zengyi Xu (徐增熠) ${ }^{1,2}$, Wenqing Niu (牛文清) ${ }^{1}$, Jianyang Shi (施剑阳) $)^{1}$, and Nan Chi (迟 楠) ${ }^{1 *}$ \\ ${ }^{1}$ Key Laboratory for Information Science of Electromagnetic Waves (MoE), Department of Communication Science and Engineering, Fudan University, \\ Shanghai 200433, China \\ ${ }^{2}$ Peng Cheng Laboratory, Shenzhen 518055, China
}

*Corresponding author: nanchi@fudan.edu.cn

Received November 26, 2021 | Accepted January 25, 2022 | Posted Online February 18, 2022

\begin{abstract}
In this paper, we propose a 36-quadrature amplitude modulation (QAM] superposition modulation technique that is featured with uneven symbol probability by nonlinear precoding, named nonlinear coded nonuniform superposition (NCNS) QAM. Its aim is to alleviate the nonlinearity effect caused by high instantaneous power in multi-input single-output (MISO) visible light communication (VLC) system, with an uneven probabilistic-shaped constellation. The transmitter includes two LEDs to send signals independently, and the receiver uses a photo detector to receive the superposed QAM signal. The experiment results show that NCNS has a better robustness against nonlinearity than pulse amplitude modulation 4, approximately gaining a $16 \%$ increase in maximum usable peak-to-peak voltage and a $33 \%$ enlargement in dynamic range area. It is a simple but effective approach to solve the bandwidth limits related to signal power and hopefully be applied in large power VLC systems such as underwater VLC, or to improve the robustness against power fluctuation.
\end{abstract}

Keywords: visible light communication; light-emitting diode; multi-input single-output; quadrature amplitude modulation; nonlinear coding; spectral shaping.

DOI: 10.3788/COL202220.042501

\section{Introduction}

The visible light communication (VLC) system, which utilizes the $400-800 \mathrm{THz}$ spectrum, is predicted as a promising technique in the upcoming $6 \mathrm{G}$ communication due to its desired characteristics such as high confidentiality, rich spectrum resources, being free from license and patent, low electromagnetic pollution, and other advantages ${ }^{[1]}$.

The multi-input multi-output (MIMO) system has proven its importance in the past $4 \mathrm{G}$ and current $5 \mathrm{G}$ networks ${ }^{[2]}$. It solves the demanding challenge for communication capacity and provides large-scale access for massive users. Its single-output version is termed multi-input single-output (MISO). It is believed that this technology would continue to play a crucial role in $6 \mathrm{G}$ to implement a more massive connectivity ${ }^{[3,4]}$, which paves the way to an 'Internet of Everything' (IoE) network.

This situation encourages the combination of VLC with MIMO/MISO, where LEDs and photo detector (PD) arrays resemble the antennas in their RF counterparts. The bandwidth limitation of LEDs can be compensated by increasing the number of parallel transmitters ${ }^{[5]}$. Meanwhile, compared with
RF system, it has a potential to be integrated into indoor illuminating systems. Existing works ${ }^{[6-9]}$ have explored the application of MIMO/MISO in VLC systems. A common point in these works is that independent signals are superposed at the same receiver. However, the nonlinearity effect is a severe issue in $\mathrm{VLC}^{[10]}$. Apart from the nonlinearity from the LED transmitter, the instantaneous photo voltage on the PD at the receiver is more likely to enter the nonlinear region due to the multi-fold incident light power. The degraded response characteristic deteriorates the communication quality and raises the bit error rate (BER) above the threshold for forward error correction (FEC).

Nonlinear coding is an effective measure to alleviate the negative influence from nonlinearity, which is able to modify the signal in either the time domain or frequency domain (or both), in the meantime, as some of them apply trellis-coded modulation (TCM) that adds correlation between adjacent symbols $^{[11-14]}$. Thus, their symbols appear in a nonuniform probability distribution. In this paper, we are inspired by Yamamoto et al. in Refs. $[15,16]$, where they present a trelliscoded nonlinear precoding technique, which also changes the 
probability distribution. But unlike probabilistic shaping (PS), it does not require a distribution matcher (DM) or add redundancy in the transmitted sequence ${ }^{[17]}$. Meanwhile, its spectrum shows a prominent mid-frequency band. Compared with precoding using Tomlinson-Harashima precoding (THP), this algorithm consumes significantly less computation because it does not perform an estimation using previous symbols ${ }^{[18]}$.

However, the previous work in Refs. $[15,16]$ separates the transmission of two different channels using two independent receivers. With scalar superposed coded modulation (ScalarSCM), it could form a superposed quadrature amplitude modulation (QAM) signal at the receiver, which follows a similar demodulation purpose but has an unevenly distributed constellation point. Instead of using unbalanced coding schemes for different channels ${ }^{[19]}$, the two channels use an identical process of precoding. In this paper, we explored this application in an MISO VLC system and named it nonlinear coded nonuniform superposition (NCNS) QAM.

A joint least mean square (LMS) (MIMO LMS) software equalizer ${ }^{[20]}$ is utilized to compensate inter-symbol interference (ISI) and inter-channel interference (ICI) by deducting the product of the symbol value and the tap weights from the received signal. Because it is only concerned with the input, it also suits MISO systems. In this experiment, its tap weights also provide insights for ISI and ICI.

\section{Methodology}

Applying the algorithm shown in Fig. 1, which is described by Yamamoto et al. in Refs. [15,16], two independent six-level codes $c^{6}(n)$ are obtained from two uniformly distributed fourlevel signals $c^{4}(n)$. It leaves a clear path in the trellis diagram. The distribution of $c^{6}(n)$ can be written in the following form:

$$
\boldsymbol{P}=\left[P_{0}, P_{1}, P_{2}, P_{3}, P_{4}, P_{5}\right]^{T}=\left[\frac{1}{16}, \frac{3}{16}, \frac{4}{16}, \frac{4}{16}, \frac{3}{16}, \frac{1}{16}\right]^{T},
$$

which is the eigenvector corresponding to the unity eigenvalue of the transition matrix. Two independent channels would result in a $2 \mathrm{D}$ constellation with nonuniform distribution of probability:

$$
\boldsymbol{P}_{X}=\boldsymbol{P P}^{T}=\left[\begin{array}{cccccc}
\frac{1}{256} & \frac{3}{256} & \frac{4}{256} & \frac{4}{256} & \frac{3}{256} & \frac{1}{256} \\
\frac{3}{256} & \frac{9}{256} & \frac{12}{256} & \frac{12}{256} & \frac{9}{256} & \frac{3}{256} \\
\frac{4}{256} & \frac{12}{256} & \frac{16}{256} & \frac{16}{256} & \frac{12}{256} & \frac{4}{256} \\
\frac{4}{256} & \frac{12}{256} & \frac{16}{256} & \frac{16}{256} & \frac{12}{256} & \frac{4}{256} \\
\frac{3}{256} & \frac{9}{256} & \frac{12}{256} & \frac{12}{256} & \frac{9}{256} & \frac{3}{256} \\
\frac{1}{256} & \frac{3}{256} & \frac{4}{256} & \frac{4}{256} & \frac{3}{256} & \frac{1}{256}
\end{array}\right],
$$

where $\boldsymbol{P}_{X}$ is the probability matrix of the combined vector symbol at the receiver. $\boldsymbol{P}_{X_{i, j}}=P_{i} \cdot P_{j}, X_{i, j}$ represent the case when channel 1 transmits the $i$ th six-level signal and channel 2 transmits the $j$ th.

To implement the vector symbol in an MISO system, firstly, the positive integers in $c^{6}(n)$ are shifted and scaled into $c^{\prime 6}(n) \in$ $\{-5,-3,-1,1,3,5\}$ as pulse amplitude modulation (PAM) requires. It needs upsampling and a roll-off filtering process to achieve pulse shaping:

$$
\left[\begin{array}{l}
c_{1}(t) \\
c_{2}(t)
\end{array}\right]=\left[\begin{array}{l}
g(t) \otimes c_{1}^{\mathrm{up}}(t) \\
g(t) \otimes c_{2}^{\mathrm{up}}(t)
\end{array}\right]
$$

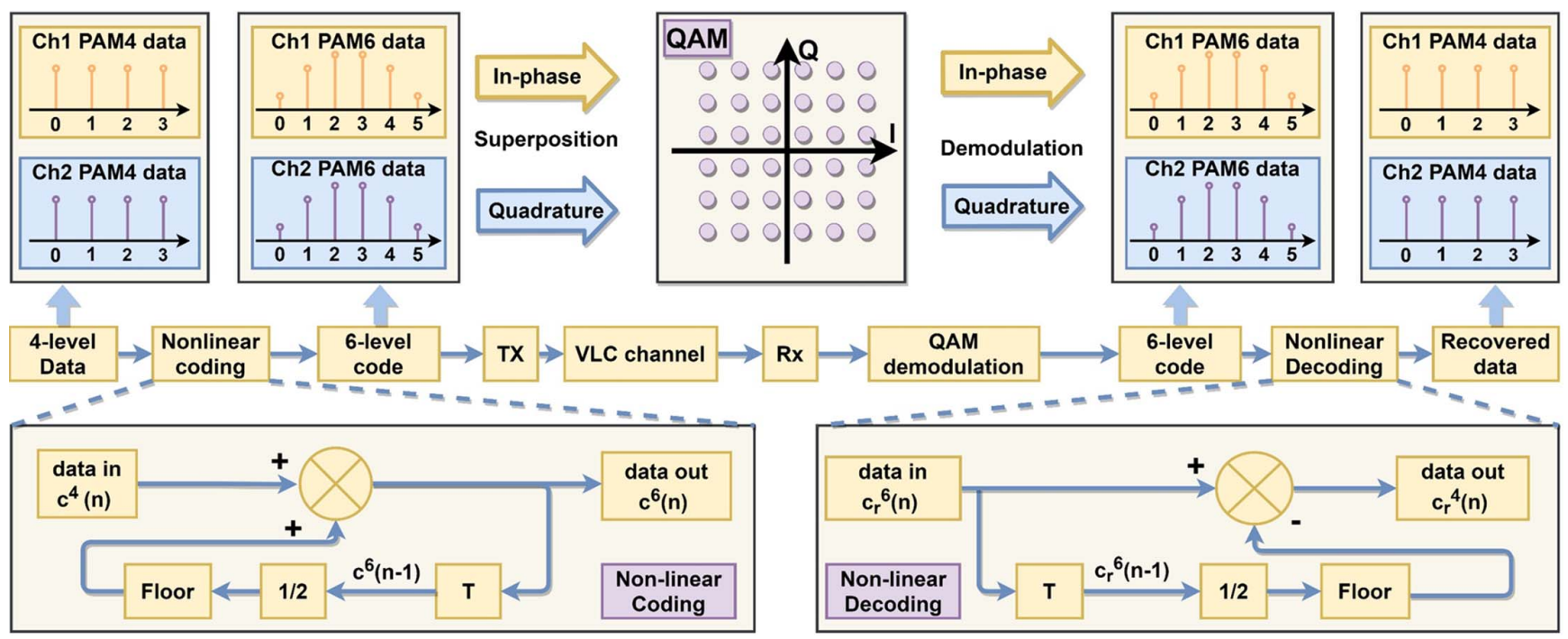

Fig. 1. Principle of NCNS-QAM algorithm. A 36-QAM is implemented by superposing two independent PAM6 signals generated by the original PAM4 ones. The inphase and quadrature signals are later demodulated as in the regular QAM scheme, resulting in two independent PAM6 data. After the nonlinear decoding step, the original PAM4 data is recovered. 
where $c^{\text {up }}(t)$ is the upsampled six-level code from $c^{\prime 6}(n)$, and $c(t)$ is the result from the filter.

Next, the filtered samples are mixed with one of the two orthogonal carriers, either $\sin \left(2 \pi f_{0} t\right)$ or $\cos \left(2 \pi f_{0} t\right)$, as the equation describes:

$$
\left\{\begin{array}{l}
\operatorname{PAM}_{1}(t)=c_{1}^{\prime}(n) \cdot \cos \left(2 \pi f_{0} t\right) \\
\operatorname{PAM}_{2}(t)=c_{2}^{\prime}(n) \cdot \sin \left(2 \pi f_{0} t\right)
\end{array}\right.
$$

The expression for the two PAM signals reminds us of the description of the in-phase and quadrature component in QAM. One pair of superposed orthogonal signals can be regarded as a single complexed-valued QAM symbol:

$$
s \mathrm{QAM}(t)=\operatorname{PAM}_{1}(t)+j \mathrm{PAM}_{2}(t)
$$

which can be written in a complex-valued expression:

$$
s \operatorname{QAM}(t)=\boldsymbol{X}(n) \cdot e^{j\left(2 \pi f_{0} t\right)},
$$

where $\boldsymbol{X}(n)=c_{1}^{\prime}(n)+j c_{2}^{\prime}(n)$. Thus, the input scalar symbol is already converted into a vector symbol. However, the probability of each symbol is not equal due to the uneven distribution of $c^{\prime}(t)$.

To decode the 36-QAM signals, the process is similar to that in QAM:

$$
\left[\begin{array}{l}
R_{1}(t) \\
R_{2}(t)
\end{array}\right]=\left[\begin{array}{cc}
\cos \left(2 \pi f_{0} t\right) & 0 \\
0 & -\sin \left(2 \pi f_{0} t\right)
\end{array}\right]\left[\begin{array}{l}
\operatorname{sQAM}(t) \\
\operatorname{sQAM}(t)
\end{array}\right]
$$

The result experiences down sampling and another round of roll-off filtering with the same parameter as in Eq. (3) to obtain the recovered data $R_{\text {rec }_{1}}$ and $R_{\text {rec }_{2}}$ :

$$
\left[\begin{array}{l}
R_{\mathrm{rec}_{2}}(t) \\
R_{\mathrm{rec}_{1}}(t)
\end{array}\right]=\left[\begin{array}{l}
g(t) \otimes R_{1}^{\text {down }}(t) \\
g(t) \otimes R_{2}^{\text {down }}(t)
\end{array}\right] .
$$

The recovered data is later processed using maximum likelihood decision (or minimum Euclidian distance). The result is the six-level codes $c_{r_{1}}^{6}(n)$ and $c_{r 2}^{6}(n)$. By applying the nonlinear decoding algorithm illustrated in Fig. 1, the four-level recovered data would be obtained.

This precoding technique has a linear time complexity $O(n-$ 1) to the length $n$ of the original four-level code and a fixed space complexity $O(1)$ as the memory only stores the last six-level code and the current four-level one to encode a new six-level one.

The experiment setup is illustrated in Fig. 2. The input data is firstly encoded into PAM4 and afterwards mapped into PAM6. The signal is upsampled by three times and experiences a square-root cosine roll-off filter to control its spread in time domain. The up-conversion step divides the signal into in-phase and quadrature components and feeds them into the two independent channels in the arbitrary waveform generator (AWG). Simultaneously, an offline file is stored for synchronization.
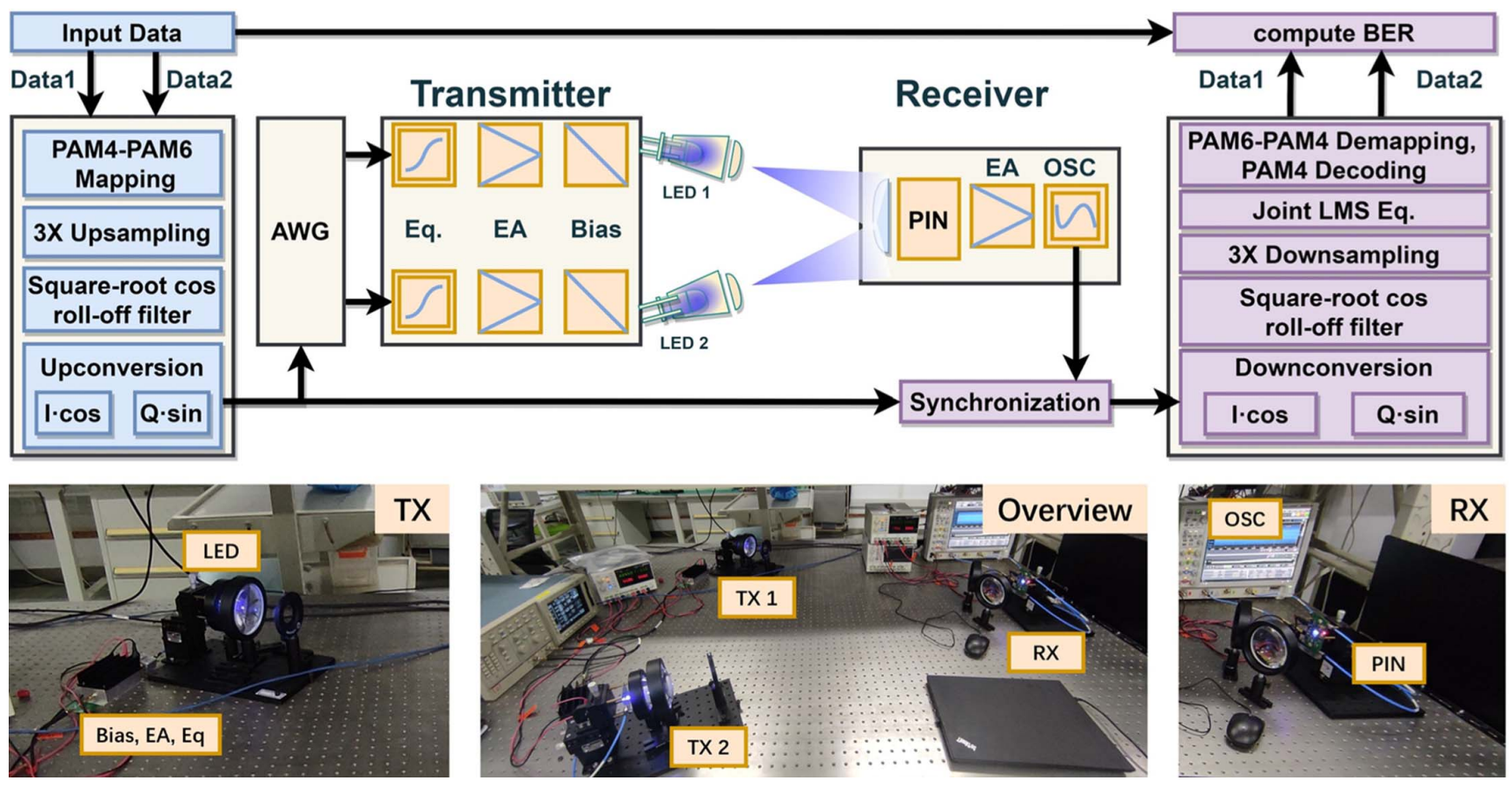

Fig. 2. Setup for the NCNS-QAM experiment. Two independent LED channels transmit optical signals, which are received by a single PIN detector. The original fourlevel PAM4 signal experiences nonlinear coding (PAM4 to PAM6 mapping), upsampling, roll-off filtering, before up-conversion. In up-conversion, the two channels are mixed with orthogonal carriers and thus form a QAM signal at the receiver. After the signal is received, it undergoes down-conversion (as in QAM demodulation), roll-off filtering, joint LMS equalization, and nonlinear decoding. TX, transmitter; RX, receiver; Eq., hardware equalizer; EA, electrical amplifier; OSC, oscilloscope; PIN, p-i-n photo detector. 
Next, the signal is equalized, amplified, and biased for the transmission using LEDs. The received signal is sampled from the oscilloscope and experiences the inverse process it has been through in the transmitter. An extra step, LMS software equalization, is added to compensate the linear loss in the channel. To improve the BER performance, the LMS algorithm is applied at the receiver. Its taps weights provide evidence to compare the performance of PAM4 and NCNS. Finally, by comparing the decoded information to the original data saved in an offline file, the BER is computed and used for evaluation of the communication quality.

The MISO system used in this experiment is built using two LEDs and one PIN photodiode. The LED used is developed by Nanchang University, featuring $450 \mathrm{~nm}$ peak wavelength and maximum $2.25 \mathrm{~Gb} / \mathrm{s}$ data rate. The PIN is Hamamatsu S10784, of which the usable wavelength covers $400-1000 \mathrm{~nm}$. The oscilloscope is Agilent Technologies MSO9254A, and the AWG is SONY Tektronix AWG520. The bias tee and amplifier used in this experiment are Mini-Circuits ZFBT-4R2GW-FT and ZHL-6 A-S+.

\section{Result}

This experiment tests both the PAM4 and NCNS modulation schemes, setting the bias current at $35 \mathrm{~mA}$. The AWG generates the transmitted signal at $900 \mathrm{MSa} / \mathrm{s}$ with three times upsampling. The data rate in this experiment is therefore $1200 \mathrm{Mb} / \mathrm{s}$. To demonstrate and compare the strength and weakness of the two modulation schemes, this experiment samples the BER of both at varying $V_{\mathrm{pp}}$ from $200 \mathrm{mV}$ to $1000 \mathrm{mV}$, which includes both the low signal-to-noise case and the high $V_{\text {pp }}$ (also high nonlinearity) case.

The samples constitute two five-by-five grids, whose diagonal line shows the case where the two channels are balanced with equal $V_{\mathrm{pp}}$ (as the baseline of analysis). The result is plotted in Fig. 3. The line chart in Fig. 3 shows the case where both

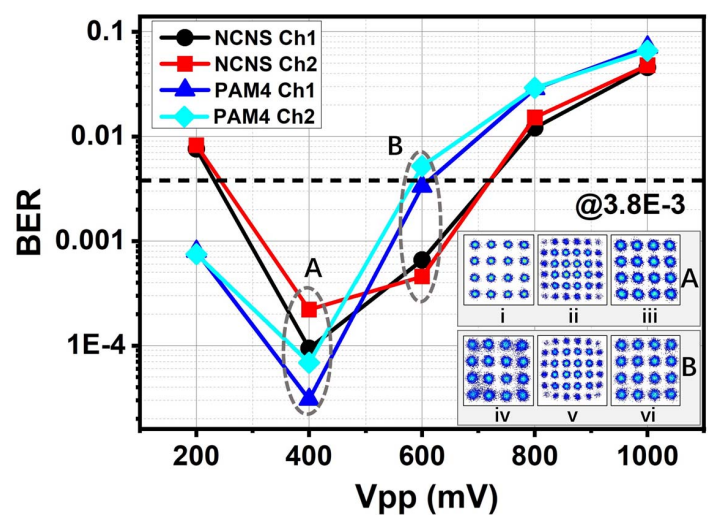

Fig. 3. BER versus $V_{p p}$ for both PAM4 and NCNS. The A group insets are the constellation diagrams at $400 \mathrm{mV}$ : (i) PAM4, (ii) NCNS, and (iii) the four-level decoded result from NCNS. The B group insets are the constellation diagrams at $600 \mathrm{mV}$ : (iv) PAM4, (v) NCNS, and (vi) the four-level decoded result from NCNS. channels transmit signals with equal $V_{\mathrm{pp}}$. Although, in the low power case below $600 \mathrm{mV} V_{\mathrm{pp}}$, NCNS is outperformed by PAM4, the maximum usable $V_{\mathrm{pp}}$ of NCNS is about $100 \mathrm{mV}$ (or $16 \%$ ) higher than that of PAM4. The insets give more details about this phenomenon. In each group, the three diagrams from left to right indicate the signal quality of PAM4, NCNS, and the result from NCNS decoding, intuitively showing the effect of NCNS.

In the $400 \mathrm{mV}$ case, because the distance between the constellation points in NCNS is less than that in PAM4, and SNR is comparatively low, the constellation points in NCNS are less desirable compared with those in PAM4, so are the constellation points after nonlinear decoding in NCNS. However, the situation is flipped in the $600 \mathrm{mV}$ case, where the difference in BER is the most significant. The nonlinearity remarkably distorts the PAM4 constellation points, especially the peripheral ones. For example, the constellation points at the four corners are so severely distorted that they lose their round shape.

By contrast, NCNS successfully limits the deformation of constellation points. Meanwhile, the decoded result also demonstrates more concentrated constellation points compared with PAM4. The constellation diagram in Fig. 3 gives an explanation to the improvement in BER by NCNS, which is due to the nonuniform distribution, and NCNS sends more signals with lower power transmitted and thus avoids the nonlinear region. Although NCNS performs less attractively when the VLC system is SNR restricted, when the nonlinearity replaces noise and becomes the main cause of BER, NCNS performs better than PAM4. This conclusion can be solidified using Fig. 4.

To provide detailed information, the power spectra at $400 \mathrm{mV}$ and $600 \mathrm{mV} V_{\mathrm{pp}}$ are plotted in Fig. 4. The histogram of symbols from single channel and after the superposed QAM is also plotted in Fig. 4. Compared with the PAM4 spectrum in Fig. 4(a), the spectrum in Fig. 4(e) is less distinguishable from the background noise. However, at $600 \mathrm{mV}$, with the SNR increased in both spectra in Figs. 4(b) and 4(f), the signal bands are clearly distinguishable from background noise. Figures 4(c) and 4(g) illustrate the distribution of the received symbols, where PAM4 is mainly uniform, while NCNS is otherwise. The last pair of diagrams in Figs. 4(d) and 4(h) shows the distribution of the superposed QAM signal by PAM4 and by NCNS, which also demonstrates their difference in symbol probability. Furthermore, in Fig. 4(c), the histogram suggests that peaks are overlapping except for the central two, while in Fig. 4(g) this phenomenon is less severe. It means that more received symbols are distributed closer to the expected value in NCNS than in PAM4, which means fewer symbols are wrongly decided as it deviates from its original value and falls into the decision region for other symbols.

Assuming the averaged amplitude on each tap of the joint LMS equalizer is the same, the tap weight indirectly gives information about the intensity of ISI and ICI. The result is plotted in Fig. 5, where Fig. 5(a) gives the tap weights $w_{11}$ for ISI, and Fig. 5(b) shows tap weights $w_{12}$ for ICI. The RMS value of the tap weights is calculated and recorded in Table 1. To further explore the details, the RMS value and the ratio of the center tap weight 


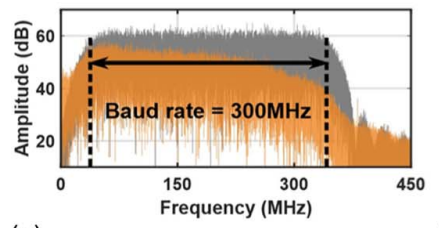

(a)

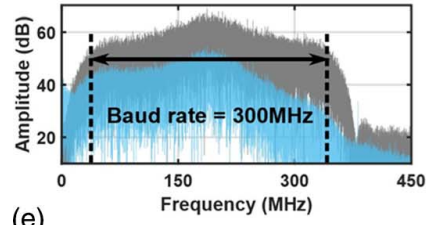

(e)

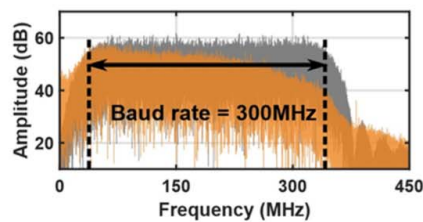

(b)

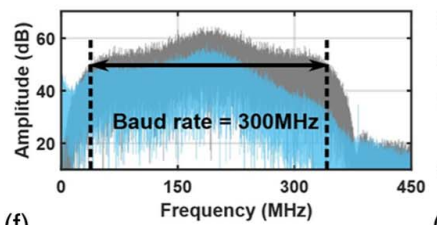

(f)

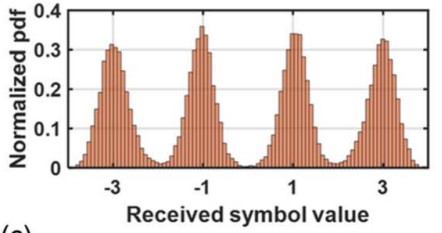

(c)

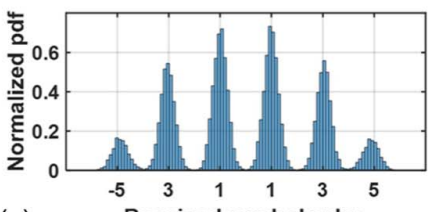

(g)

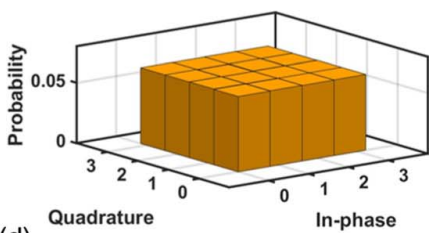

(d)

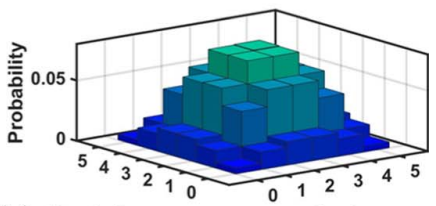

(h) Quadrature

In-phase

Fig. 4. First and second columns are the spectra of $T X$ and RX signals in both PAM4 (orange) and NCNS (blue). (a), (e) $V_{p p}=400 \mathrm{mV}$ and (b), ( $f$ ) $V_{p p}=600 \mathrm{mV}$. The third column is the probability density of RX symbol under $600 \mathrm{mV}$ in (c) PAM4 and (g) NCNS. The last column is the probability distribution of each symbol in superposed QAM by (d) PAM4 or (h) NCNS when transmitting the same length of original data.

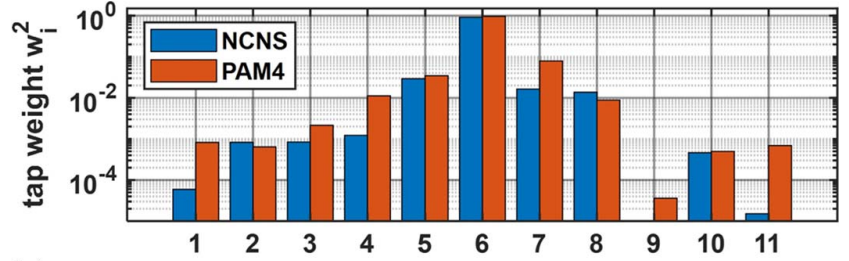

(a) LMS tap index

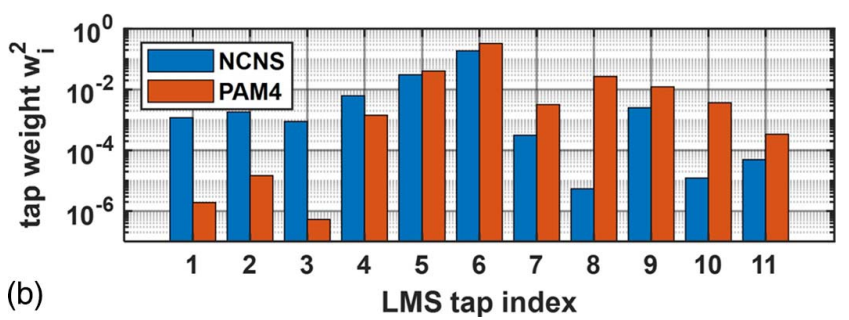

Fig. 5. Joint LMS equalizer tap weights in channel 1. (a) shows the tap weights $W_{11}$ for compensating inter-symbol interference (ISI) from channel 1 itself, and (b) shows the tap weights $w_{12}$ for compensating inter-channel interference (ICI) from channel 2. The two channels are assumed symmetrical in ISI and $\mathrm{ICl}$. The data are collected at $V_{\mathrm{pp}}=600 \mathrm{mV}$.
Table 1. ISI and ICI Measured by LMS Tap Weights.

\begin{tabular}{lccccc}
\hline Channel/Code & $W_{0}$ & RMS ISI & RMS ICI & ISI $/ W_{0}$ & ICI $/ W_{0}$ \\
\hline CH1/NCNS & 0.956 & 0.0755 & 0.145 & $7.90 \%$ & $15.2 \%$ \\
CH2/NCNS & 0.921 & 0.0829 & 0.137 & $9.00 \%$ & $14.9 \%$ \\
CH1/PAM4 & 0.981 & 0.1120 & 0.195 & $11.4 \%$ & $19.8 \%$ \\
CH2/PAM4 & 0.984 & 0.1120 & 0.206 & $11.3 \%$ & $20.9 \%$ \\
\hline
\end{tabular}

$w_{0}$ to the RMS value of the others are calculated and listed in the Table 1, where both channels in NCNS and PAM4 are included. NCNS presents a lower ratio in all of the four cases.

As the LMS equalizer outputs the weighted sum of all of the taps, the squared weight can be interpreted as the influence in signal power from the adjacent symbols, assuming the averaged value from each tap is the same. The central tap (tap 6) in Fig. 5(a) corresponds to the influence from the expected symbol, but the others are regarded as ISI and ICI. The data in Table 1 suggests that, in the NCNS case, the LMS output is dominated by
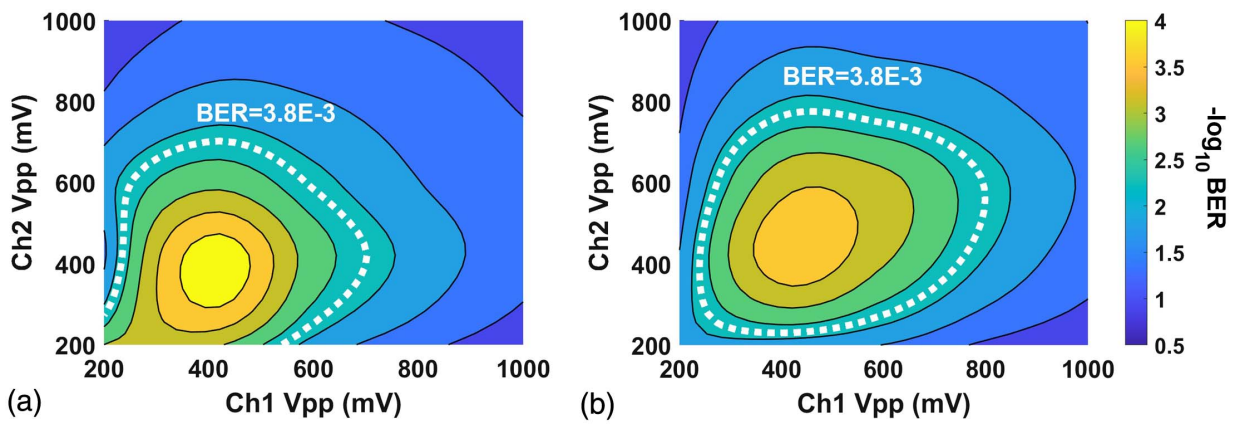

Fig. 6. Comparison in averaged BER of the two channels between the (a) PAM4 and (b) NCNS. The area enclosed by the dash line represents the usable area with BER lower than the threshold for $7 \%$ FEC. NCNS has $0.24 \mathrm{~V}^{2}$ dynamic range, which is $33 \%$ larger than that of PAM $4\left(0.18 \mathrm{~V}^{2}\right)$. 
the expected symbol and less affected by the adjacent one or from the other channel.

Figure 6 plots two heat maps of the mean BER of the two channels. The result is generated from interpolation to the five-by-five grid of the original 25 samples using the cubic function. It gives a distinct contrast in dynamic area enclosed by the threshold BER level. The subplot of Figs. 6(a) and 6(b) shows that NCNS provides a larger dynamic range for lossless communication with 7\% FEC applied, extending the boundary into the right top corner where the nonlinearity is the most severe. The gradient in the color map indicates that NCNS has a flatter BER distribution as Fig. 3 also shows. The dynamic area in NCNS is $2.40 \times 10^{5} \mathrm{mV}^{2}$, about $33 \%$ larger than that in PAM4 $\left(1.8 \times 10^{5} \mathrm{mV}^{2}\right)$. The final result in Fig. 6 clearly demonstrates the merit of NCNS. Compared with superposed modulation using PAM4, the maximum usable range increases by $33 \%$. This improvement means that higher signal power could be applied in the VLC system. Meanwhile, the system is more robust against fluctuating $V_{\mathrm{pp}}$. When the bias point is the same, NCNS allows a larger jittering in $V_{\mathrm{pp}}$ but still keeps the BER under the threshold.

\section{Conclusion}

The distribution of symbols plays a decisive role in their performance within the same $V_{\mathrm{pp}}$, which accounts for most of the difference it presents compared with PAM4. In this experiment, NCNS precoding lowers the averaged signal power and thus increases the dynamic range significantly in high $V_{\mathrm{pp}}$, but it sacrifices the system performance at lower $V_{\mathrm{pp}}$. This feature is desirable in large power VLC systems, where the high power frequently causes severe nonlinearity. Apart from terrestrial VLC networks, underwater VLC (UVLC) systems would especially benefit, as the power requirement is usually higher to overcome the scattering and absorption. In the meantime, the VLC system could be more robust when jittering in $V_{\mathrm{pp}}$ is present. With NCNS and its larger dynamic range, the $V_{\mathrm{pp}}$ is less likely to cross the boundary, and thus the VLC system is more possible to control BER under the threshold. This experiment shows that NCNS-QAM could be a useful modulation scheme in the future and help the VLC system to stretch further and deeper into the unknown region.

\section{Acknowledgement}

This work was partially supported by the National Natural Science Foundation of China (Nos. 61925104, 62031011, and 62074072), China National Postdoctoral Program for Innovative Talents (No. BX2021082), China Postdoctoral Science Foundation (No. 2021M700025), and Major Key Project of PCL (No. PCL2021A14).

\section{References}

1. N. Chi, Y. Zhou, Y. Wei, and F. Hu, "Visible light communication in 6G: advances, challenges, and prospects," IEEE Veh. Technol. Mag. 15, 93 (2020).

2. W. Jiang, B. Han, M. A. Habibi, and H. D. Schotten, "The road towards 6G: a comprehensive survey," IEEE Open J. Commun. Soc. 2, 334 (2021).

3. S. Chen, J. Zhang, Y. Jin, and B. Ai, "Wireless powered IoE for 6G: massive access meets scalable cell-free massive MIMO," China Commun. 17, 92 (2020).

4. Y. L. Lee, D. Qin, L. C. Wang, and G. H. Sim, "6G massive radio access networks: key applications, requirements and challenges," IEEE Open J. Veh. Technol. 2, 54 (2021).

5. C. Chen, W. Zhong, H. Yang, and P. Du, "On the performance of MIMONOMA-based visible light communication systems," IEEE Photonics Technol. Lett. 30, 307 (2018).

6. W. Wang, Y. Zhu, Y. Zhang, and J. Zhang, "An optimal power allocation for multi-LED phase-shifted-based MISO VLC systems," IEEE Photonics Technol. Lett. 27, 2391 (2015).

7. H. Ji, S. Qiao, and T. Zhang, "A MISO-VLC system based on LACO-OFDM and superposed constellation demodulation," in 9th International Conference on Information Science and Technology (2019), p. 294.

8. L. Qiao, X. Lu, S. Liang, J. Zhang, and N. Chi, "MISO visible light communication system utilizing hybrid post-equalizer aided pre-convergence of STBC decoding," Chin. Opt. Lett. 16, 060604 (2018).

9. C. Wang, G. Li, F. Hu, Y. Zhao, J. Jia, P. Zou, Q. Lu, J. Chen, Z. Li, and N. Chi, "Visible light communication for vehicle to everything beyond $1 \mathrm{~Gb} / \mathrm{s}$ based on an LED car headlight and a $2 \times 2$ PIN array," Chin. Opt. Lett. 18, 110602 (2020).

10. X. Deng, S. Mardanikorani, Y. Wu, K. Arulandu, B. Chen, A. M. Khalid, and J. M. G. Linnartz, "Mitigating LED nonlinearity to enhance visible light communications," IEEE Trans. Commun. 66, 5593 (2018).

11. A. R. Calderbank, T. Lee, and J. E. Mazo, "Baseband trellis codes with a spectral null at zero," IEEE Trans. Inf. Theory 34, 425 (1988).

12. K. Igarashi, T. Tsuritani, and I. Morita, "Polybinary shaping for highly-spectral-efficient super-Nyquist WDM QAM signals," J. Lightwave Technol. 34, 1724 (2016).

13. J. S. Park, S. B. Gelfand, and M. P. Fitz, "A spectral shaping nonlinear binary coded modulation with Gray-mapped QAM signals," in Milcom 2010 Military Communications Conference (2010), p. 2363.

14. N. Stojanovic, C. Prodaniuc, F. Karinou, and Z. Qiang, "56-Gbit/s 4-D PAM4 TCM transmission evaluation for $400-\mathrm{G}$ data center applications," in Optical Fiber Communications Conference and Exhibition (2016), paper Th1G.6.

15. S. Yamamoto, A. Masuda, H. Taniguchi, and Y. Kisaka, "92-Gbaud PAM4 transmission using spectral-shaping trellis-coded-modulation with 20GHz bandwidth limitation," in Optical Fiber Communications Conference and Exhibition (2019), paper W4I.5.

16. S. Yamamoto, H. Taniguchi, A. Matsushita, M. Nakamura, S. Okamoto, and Y. Kisaka, "Spectral-shaping technique based on nonlinear-codedmodulation for short-reach optical transmission," J. Lightwave Technol. 38, 466 (2020)

17. F. Buchali, F. Steiner, G. Böcherer, L. Schmalen, P. Schulte, and W. Idler, "Rate adaptation and reach increase by probabilistically shaped 64-QAM: an experimental demonstration," J. Lightwave Technol. 34, 1599 (2016).

18. H. Xin, K. Zhang, D. Kong, Q. Zhuge, Y. Fu, S. Jia, W. Hu, and H. Hu, "Nonlinear Tomlinson-Harashima precoding for direct-detected double sideband PAM-4 transmission without dispersion compensation," Opt. Express 27, 19156 (2019).

19. X. Guo and N. Chi, "Superposed 32QAM constellation design for $2 \times 2$ spatial multiplexing MIMO VLC systems," J. Lightwave Technol. 38, 1702 (2020).

20. H. Huang, P. Shih, C. Lin, Y. Cheng, W. Liang, C. Ho, C. Wei, and A. Ng'oma, " $2 \times 2$ MIMO OFDM-RoF system employing LMS-based equalizer with I/Q imbalance compensation at $60 \mathrm{GHz}$," IEEE Photonics J. 6, 7200307 (2014). 